\title{
Multi-temporal active power scheduling and voltage/var control in autonomous microgrids
}

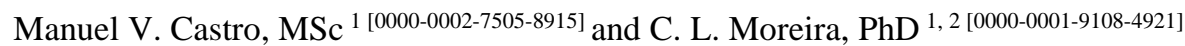 \\ ${ }^{1}$ INESC TEC (Institute for Systems and Computer Engineering, Technology and Science) \\ Porto, Portugal \\ ${ }^{2}$ University of Porto, Faculty of Engineering \\ Porto, Portugal \\ manuel.v.castrodinesctec.pt \\ carlos.moreira@inesctec.pt
}

\begin{abstract}
This paper presents a multi-temporal approach for the energy scheduling and voltage/var control problem in a microgrid (MG) system with photovoltaic (PV) generation and energy storage devices (PV-battery MG) during islanded operation conditions. A MG is often defined as a low voltage (LV) distribution grid that encompasses distributed energy resources and loads that operate in a coordinated way, either connected to the upstream distribution grid or autonomously (islanded from the main grid). Considering the islanded operation of the MG during a given period, it is necessary to develop proper tools that allow the effective coordination of the existing resources. Such tools should be incorporated in the MG control system hierarchy in order to assure proper conditions for the operation of the autonomous MG in terms of active power, voltage and reactive power management. Energy storage devices are essential components for the successful operation of islanded MG. These devices have a very fast response and are able to absorb/inject the right amount of power. For the operation of the MG in islanding conditions during a longer period, it is necessary to integrate information related to the forecasting of loads and PV-based generation for the upcoming hours for which is intended to maintain MG in islanded operation. Therefore, this paper presents a tool to be integrated in the Microgrid Central Controller (MGCC) that is responsible to perform a multi-temporal optimal power flow $(\mathrm{OPF})$ in order to schedule the active and reactive power within the MG for the next time intervals.
\end{abstract}

Keywords: Microgrid; voltage/var control; storage dispatch; renewable energy integration; autonomous operation.

\section{Introduction}

Environmental concerns, political instability in fossil fuels exporting countries and a rapid technological development on energy conversion systems are the main factors for a rapidly integration of small-scale Renewable Energy Sources (RES) in the structure of power distribution systems [1] [2]. This lead to a paradigm shift of power systems in which generation became distributed throughout the various voltage levels and grew 
closer to the consumption points with the increasing connections of technologies such as PV panels, wind turbines, fuel cells, microturbines and combined heat and power (CHP) applications [3]-[5]. The smaller modular generation units that have lower power outputs than $100 \mathrm{~kW}$ can be denominated as Microsources (MS). They exploit RES or fossil fuels in high efficiency local CHP applications and are usually connected to the LV level. Because of the nature of their electric energy output, they are often coupled to the grid via power electronic interfaces and as consequence have very low inertia [4].

The large-scale integration of Distributed Generation (DG) based on RES in the distribution network increases the uncertainty of the power flows because of the high variability and intermittency of sources like wind and solar [3]. As consequence of the unpredictability nature of DG, there could be, in the system, sudden dips and peaks in the steady state voltage profile and branches overload. These challenges drove an implementation of multiples changes in the electrical system, especially at the LV level, to increase controllability and improve the management of several resources in order to incorporate DG without the need for massive grid reinforcements.

It is in this context that emerges the concept MG. A MG is regarded as distribution network that integrates a communication infrastructure with the aim of promoting the active management and control between MS, storage devices and loads [3]. The MG can operate in an autonomous mode for a significant period of time. This requires several features of controllability and management of storage devices and DG units in order to guarantee an adequate steady state voltage profile and no violation of technical limitations. The deployment of MG could be the key to increase the network security and resilience when extreme natural disasters or extreme weather conditions occur and prevent major blackouts because of their ability to work islanded from other grids providing a self-healing functionality [3]. The concept of the MG was studied and explored, evolving into a more general concept, which is the SmartGrid (SG) and nowadays is seen as the basic component of a SG.

Storage devices are a key component for the successful operation of autonomous power systems that do not have synchronous generators to regulate frequency. These devices are able to absorb/inject the right amount of power, if necessary in very short time [3] [6]. The operation of a PV-battery MG relies on the power-frequency and voltage-reactive power droop control concepts that can be programmed in power converters associated to energy storage devices, such that these devices can operate as a grid-forming unit, or, in other words, as a Voltage Source Inverter (VSI). During islanded operation, the primary control layer (based on the droop control) is responsible for frequency and voltage regulation strategies that will assure the continuous power balancing with minimum dependence of communication system. Then, the secondary control layer will be responsible to dispatch the available resources according to a given objective in order to restore frequency and node voltages to reference set-points [3]. Therefore, in the proposed approach, it is assumed the steady state operating conditions of the system, such that the grid frequency is at the nominal value.

The main contribution of this paper is related to the development of a multi-temporal approach for the energy scheduling and voltage/var control problem of a PV-battery MG system during islanding operating conditions. The proposed approach takes into 
consideration forecasts of PV production and load for the next time steps and performs an energy scheduling and voltage/var control problem for an islanding MG operating with several VSI coupled to energy storage devices.

\section{Microgrid concept and control architecture}

The MG concept was first introduced in the United States of America by the Department of Energy, more specifically, the Consortium for Electric Reliability Technology Solutions (CERTS) while studying the implications of DG penetration in power systems. CERTS defines the MG as being a single and self-controlled entity that supply energy to its consumers and also manages, controls and maintains the local necessities [3]. This concept was fully developed and expanded by the European Union (EU), Canada and Japan [5]. In the EU, the MG concept was designed to safely integrate the MS based RES in high levels of penetration because of environmentally concerns and economic benefits. A clear definition of a MG system, was developed by the CIGRE working group WG C6.22 following concept: "MicroGrids are electricity distribution systems containing loads and distributed energy resources, (such as distributed generators, storage devices, or controllable loads) that can be operated in a controlled, coordinated way either while connected to the main power network or while islanded" [7].

The operation of a MG requires high levels of flexibility and control. For this purpose, it was essential to establish a hierarchical management and control scheme supported by a communication infrastructure. In the higher level of MG management and control is the Microgrid Central Controller (MGCC) located in LV side of the MV/LV transformer. The MGCC communicates with the lower level controllers and is responsible for a proper technical and economic management of the MG. The secondary hierarchy control is located near each of controllable MS, storage devices and controllable loads (Fig. 1). The controllers of MS and storage are called Microsource controllers (MC) and usually are housed in within power electronic interface of the MS. The controllable loads or groups are regulated and adjusted by load controllers (LC). The MGCC provides power set-points to the secondary controllers (LC and MC) according to a pre-defined strategy. The MC responds in milliseconds to the demands from MGCC and from local information and have autonomy to optimize local generation, during interconnected mode, and fast load following, during islanding of the MG. The LC responds to demands of the MGCC like implement load-shedding features during emergency circumstances. It is also the information available in the MC and LC terminals that indirectly determined a set of operation and control actions that guarantee the stability of the system during transitional periods [4]. 


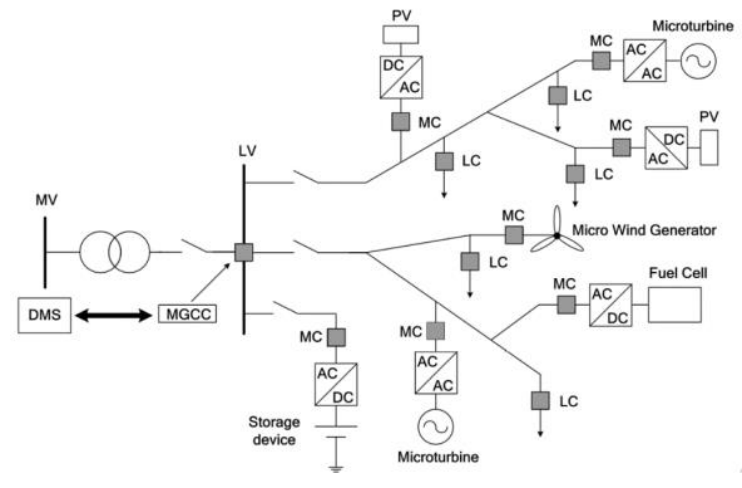

Fig. 1. MG architecture [4].

\subsection{Microgrid operation modes}

Through the exploitation of its control and management system, the MG can operate in two distinct modes: normal interconnected mode and autonomous/islanded operation mode. These modes of operation are briefly addressed hereafter.

Normal interconnected mode. In interconnected mode, the MG is connected to the medium voltage network (MV) and can partially or totally be supplied by the MV and inject the exceeding power in the MV [6]. This mode of operation can have different objectives: minimize the costs of operation or maximize revenue (economical), maintain voltage levels and/or minimize branch losses (technical) and reduce $\mathrm{CO} 2$ emissions (environmental) [3]. In this mode, the MG is operated and controlled by the MGCC that communicates with Distribution Management System (DMS). The DMS is located upstream, in the distribution network, and the MGCC is installed in the LV side of the MV/LV transformer (see Fig. 1) [5]. The DMS enables the possibility of information exchange and establishment of contractual agreements between the MG and the Distribution System Operator (DSO). The MG can be seen as an aggregation of loads and MS operating as a single entity in order to simplify their interaction with the DSO.

Islanded or emergency operation mode. The islanded or emergency mode (also known as autonomous) can be triggered by a disturbance in the upstream network or by a planned action. The disturbance in the network can be as simple as a line fault to an event like a tornado or an earthquake that severely damages electrical equipments of the power system and can cause a general blackout. A planned action of disconnection can be executed in order to perform maintenance or reparations on the system. When a MG is disconnected from the MV network, operates autonomously in a similar way to the electric power systems of the physical islands [6]. In this mode, the stability of the MG is assured by the coordination between the MGCC and the MC and LC [3]. The disconnection from the MV network requires an instant change in the output power control of MC, switching from power dispatch mode to a frequency and voltage control 
mode (primary control). Afterwards, the MGCC needs to implement a secondary control loop that performs an optimized dispatch of the MS and storage units [4]. This optimization can have as an objective to reduce system losses and maintenance of the profile of steady state bus voltage [5]. Furthermore, in case of a blackout due to an extreme event the MG can perform the local power restoration. By operating autonomously, the MG allows a reduction on interrupting times on LV levels. In addition, the MG can perform a black start, which is a service restoration that restarts the system after a blackout. This process relies on the availability of self-starting units like a battery [5].

The autonomous operation of a MG is successfully guaranteed by the implementation of adequate frequency and voltage control functionalities and after that a competent dispatch of the power reserve in the grid. These features are generally established in two levels of control: the primary and secondary control.

\subsection{Microgrid autonomous operation}

Since most of the MS connected in the MG have power electronics interfaces and there is usually not a synchronous generator installed, the primary control level of the MG (acting locally at the power electronic interface connected to the grid) depends on two fundamental types of control strategies that are available for those interfaces: PQ inverter control and VSI inverter control.

The PQ inverter control is a control mechanism that enables the power converters to be synchronized with an energized grid and it is intended to feed a given active and reactive power set point. Through this type of control, the converter operates as a current-controlled voltage source that is kept in synchronism with the grid (grid-tied inverter). Given this operational characteristic, this type of converters is usually installed in RES of energy conversion systems that has a relatively slow response to power control signals (such as microturbines) [4] [6].

The other type of control (Voltage Source Inverter (VSI), also known as a grid-forming inverter) is the one implemented for the inverters used in energy storage devices, because of their ability of acting as an independent voltage source with very fast response capabilities to load changes [6]. In principle, this control mechanism is designed by controlling voltage and frequency through the following droop functions:

$$
\begin{aligned}
& \omega=\omega_{0}-k_{P} \times P \\
& V=V_{0}-k_{Q} \times Q
\end{aligned}
$$

where,

$\omega$ - Reference angular frequency value ( $\mathrm{rad} / \mathrm{s})$.

$\omega_{0}$ - Idle value of angular frequency $(\mathrm{rad} / \mathrm{s})$ at no load conditions.

$k_{P}, k_{Q}-$ Droop slopes (rad/s.W and $\left.\mathrm{V} / \mathrm{var}\right)$.

$P$ - Inverter active power output (W)

$V$ - Reference voltage value (V).

$V_{0}$ - Idle value of voltage $(\mathrm{V})$ at no load conditions.

$Q$ - Inverter reactive power output (var). 
With this P-f droop function when there is a decrease/increase of load the frequency increases/decreases according to ( 1 ) and Fig. 2 (a). The Q-V droop function ( 2 ) and Fig. 2 (b) allows a reactive power sharing between VSI [4]. This control principle lets the VSI to react with only the information at its terminals [3].

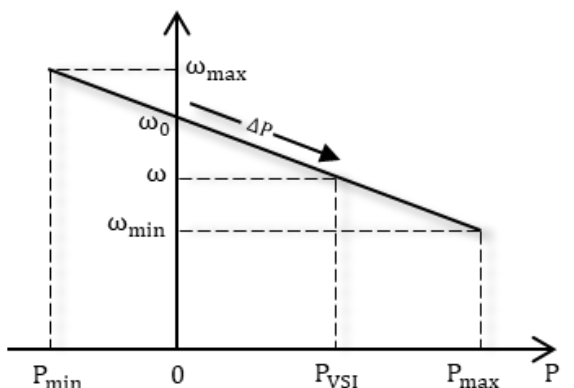

(a)

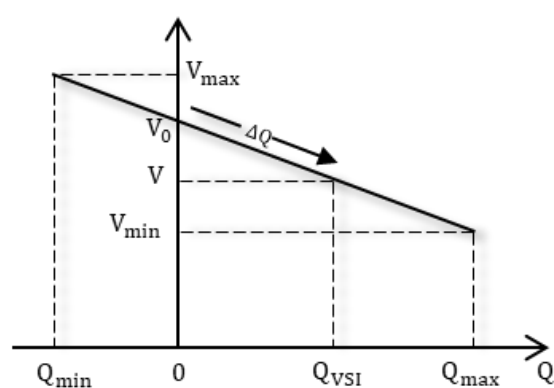

(b)

Fig. 2. VSI droop characteristics: P-f droop (a) and Q-V droop (b) [1].

In a long-term operation as an autonomous system, a MG must have a secondary control to ensure the dispatch of resources with a certain objective after the transient period from connected operation to islanded operation. The secondary control dispatches DG sending set points to the MC to compensate the frequency deviation due to the power injected by the storage devices and possibly leading to restoration of nominal frequency (or at least close enough). There were identified two secondary load-frequency strategies: one strategy implemented locally at each MC and another more centralized managed by the MGCC [3].

\section{$3 \quad$ Microgrids active and reactive power scheduling and voltage/var control during autonomous operation}

MG are typically established in LV distribution systems and the associated feeders have a predominant resistive component. As a consequence there is an important influence of the active power in the voltage profiles (while traditionally, the reactive power controls the voltage if reactance is higher than the resistance). Consequently, it is not possible to decouple the active and reactive power in the MG operation in practical terms. To overcome this issue, there are many studies and researches, such as [8], that propose the implementation of virtual impedance in the power electronic interfaces operated as VSI (grid-forming type-unit with droop control functions as previously discussed) and using the droop control concepts. The introduction of virtual impedance for droopbased controls of MG can provide mechanisms for P-Q decoupling by artificially changing the $\mathrm{X} / \mathrm{R}$ ratio through the emulation of a virtual inductor in the output impedance of VSI-type droop controlled power converters. 


\subsection{Power flow in islanded Microgrid}

The active and reactive power scheduling and voltage/var control study assumes that the frequency is controlled and maintained using the frequency control strategies mentioned previously. Therefore, the frequency is considered to remain at the nominal value $(50 \mathrm{~Hz})$. Consequently, the key concern prior to the development of any strategy is solving the power flow problem in autonomous droop-controlled MG comprise both grid-tied and grid-forming inverters (with virtual inductances).

VSI power flow model. As shown in Fig. 3, a VSI can be regarded as an AC voltage source connected to the grid through a coupling inductance. In this figure, the VSI is assumed to be connected to a LV cable (negligible inductance) being represented only the cable resistance $\left(\mathrm{R}_{\mathrm{C}}\right)$. The VSI coupling inductance corresponds to the physical one plus the virtual inductance value added to the VSI control system.

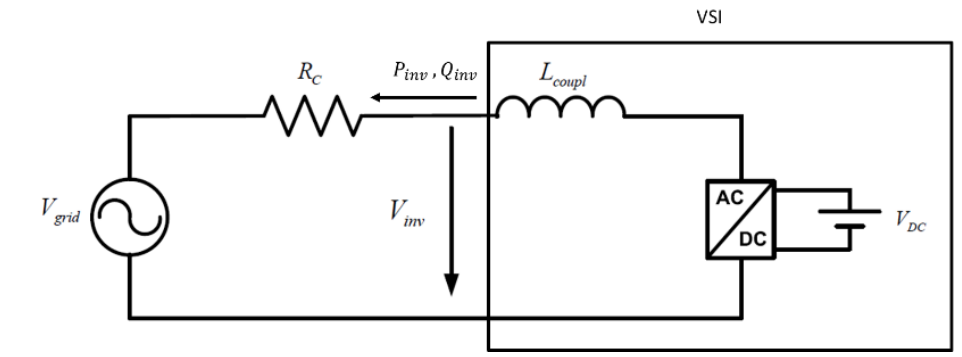

Fig. 3. VSI connected to the grid through a LV feeder [4].

The active and reactive power inverter in a LV grid can be calculated by ( 3 ) and ( 4 ).

$$
\begin{aligned}
& P_{i n v}=\frac{V_{i n v}}{R_{C}^{2}+X_{C}^{2}}\left[R_{C}\left(V_{i n v}-V_{g r i d} \cos \delta\right)+X_{C} V_{g r i d} \sin \delta\right] \\
& Q_{i n v}=\frac{V_{i n v}}{R_{C}^{2}+X_{C}^{2}}\left[R_{C}\left(V_{i n v} \sin \delta\right)+X_{C}\left(V_{i n v}-V_{g r i d} \cos \delta\right)\right]
\end{aligned}
$$

Where,

$P_{\text {inv }}$ - Inverter active power output (W).

$Q_{\text {inv }}$ - Inverter reactive power output (var).

$V_{\text {inv }}$ - Inverter terminal voltage $(\mathrm{V})$.

$R_{C}$ - Cable resistance $(\Omega)$.

$X_{C}-$ Coupling reactance $\left(\omega \cdot L_{\text {coupl }}\right)(\Omega)$.

$V_{\text {grid }}$ - Grid voltage $(\mathrm{V})$.

$\delta$ - Phase difference between inverter output voltage and grid voltage (rad).

Regarding steady state conditions, the operation of these type of inverters in LV MG includes also the "virtual inductance" concept [8] that is responsible for increasing the output inductance of the power converter and its proper operation in grids with high 
$\mathrm{R} / \mathrm{X}$ factor. Therefore, the VSI is regarded as a voltage source linked to a reactance, being represented in power flow studies using two buses (Fig. 4): an internal VSI bus and VSI output bus that acts as an interface with the grid. This model also allows a certain decoupling of active and reactive power.

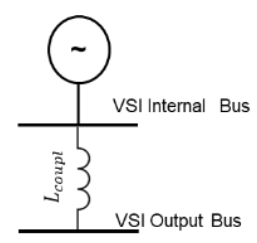

Fig. 4. VSI power flow studies model.

According to the droop equation ( 1 ), voltage control at each VSI terminal depends on the reactive power loading. Being $k_{Q}$ a fixed characteristic of each VSI, voltage and reactive power control requires the proper adjustment of the idle voltage $V_{0}$ in each unit. From the two-bus model, the idle voltage $V_{0}$ can be determined as in ( 5 ):

$$
V_{0}=V_{\text {int }}+k_{Q} \cdot Q_{\text {out }}
$$

where,

$V_{\text {int }}$ - Internal VSI bus voltage.

$Q_{\text {out }}$ - VSI reactive power output.

\subsection{A multi-temporal based approach for active and reactive power scheduling and voltage/var control}

Taking into consideration the VSI power flow model previously discussed and taking into account as well the energy capacity of the batteries, it is possible to formulate a multi-temporal based approach for active and reactive power scheduling and the voltage/var control problem to be integrated into the MGCC. This problem can be seen as an OPF based on [9] that has an objective function (OF) ( 6 ) and is subjected to the constraints ( 7 ) to ( 17 ). Equations ( 7 ) to ( 9 ) are referring to the power and energy balance for the $N$ nodes of the network. The constraints ( 10 ) to ( 17 ) refer to the technical limits of the devices or technical operation limits of the MG.

The OF considered in this formulation is the minimization of the quadratic bus voltage deviations from the nominal voltage value (1 p.u.):

$$
\min \sum_{h=1}^{H} \sum_{i=1}^{N}\left(V_{i}^{h}-1\right)^{2}
$$

subject to:

$$
P_{D G_{i}}^{h}+P_{D_{i}}^{h}-P_{C_{i}}^{h}-P_{L_{i}}^{h}=V_{i}^{h} \sum_{h=1}^{H} \sum_{k=1}^{N} V_{k}^{h} \cdot\left(\begin{array}{c}
G_{i k} \cdot \cos \left(\theta_{i}^{h}-\theta_{k}^{h}\right) \\
+B_{i k} \cdot \sin \left(\theta_{i}^{h}-\theta_{k}^{h}\right)
\end{array}\right)
$$




$$
\begin{gathered}
Q_{D G_{i}}^{h}+Q_{D_{i}}^{h}-Q_{C_{i}}^{h}-Q_{L_{i}}^{h}=V_{i}^{h} \sum_{h=1}^{H} \sum_{k=1}^{N} V_{k}^{h} \cdot\left(\begin{array}{c}
G_{i k} \cdot \sin \left(\theta_{i}^{h}-\theta_{k}^{h}\right) \\
-B_{i k} \cdot \cos \left(\theta_{i}^{h}-\theta_{k}^{h}\right)
\end{array}\right) \\
S o C_{i}^{h}=S o C_{i}^{h-1}+\left(\eta_{C_{i}} \cdot P_{C_{i}}^{h}-\frac{P_{D_{i}}^{h}}{\eta_{D_{i}}}\right) \\
V_{i}^{\text {min }} \leq V_{i}^{h} \leq V_{i}^{\max } \\
P_{D G_{i}}^{\min } \leq P_{D G_{i}}^{h} \leq P_{D G_{i}}^{\max } \\
P_{D_{i}}^{\min } \leq P_{D_{i}}^{h} \leq P_{D_{i}}^{\max } \\
P_{C_{i}}^{\min } \leq P_{C_{i}}^{h} \leq P_{C_{i}}^{\max } \\
Q_{D G_{i}}^{\min } \leq Q_{D G_{i}}^{h} \leq Q_{D G_{i}}^{\max } \\
Q_{D_{i}}^{\min } \leq Q_{D_{i}}^{h} \leq Q_{D_{i}}^{\max } \\
Q_{C_{i}}^{\min } \leq Q_{C_{i}}^{h} \leq Q_{C_{i}}^{\max } \\
\operatorname{So}_{i}^{\text {min }} \leq \operatorname{SoC}_{i}^{h} \leq \operatorname{SoC}_{i}^{\max }
\end{gathered}
$$

where,

$V_{i}^{h}$ - Voltage magnitude at bus $i$ at hour $h$

$V_{k}^{h}$ - Voltage magnitude at bus $k$ at hour $h$

$B_{i k}$ - Susceptance from bus $i$ to bus $k$

$G_{i k}$ - Conductance from bus $i$ to bus $k$

$\theta_{i}^{h}-$ Voltage angle at bus $i$ at hour $h$

$\theta_{k}^{h}-$ Voltage angle at bus $k$ at hour $h$

$P_{D G_{i}}^{h}-$ Active power output of DG $i$ at hour $h$

$P_{D_{i}}^{h}$ - Active power provided from discharging the storage unit (VSI) $i$ at hour $h$

$P_{C_{i}}^{h}$-Active power provided from charging the storage unit (VSI) $i$ at hour $h$

$P_{L_{i}}^{h}-$ Active load at the bus $i$ at hour $h$

$Q_{D G_{i}}^{h}-$ Reactive power output of DG $i$ at hour $h$

$Q_{D_{i}}^{h}$ - Reactive power provided while discharging the storage unit (VSI) $i$ at hour $h$

$Q_{C_{i}}^{h}$ - Reactive power provided while charging the storage unit (VSI) $i$ at hour $h$

$Q_{L_{i}}^{h}-$ Reactive load at bus $i$ at hour $h$

So $C_{i}^{h}$ - State of charge of VSI $i$ at hour $h$

$N$ - Total number of buses in the MG

$H$ - Total number of hours expected for MG autonomous operation

In this formulation, the control variables are the active and reactive from the storage units $\left(P_{C}, P_{D}, Q_{C}, Q_{D}\right)$, active power form PV systems $\left(P_{D G_{i}}^{h}\right)$, that will be lower than the maximum available power in case of grid technical constrains violation or violation of state of charge ( $\mathrm{SoC}$ ) conditions in energy storage devices, and the injected/absorbed reactive power in the PV panels $\left(Q_{D G}\right)$. The state variables are the voltage magnitude and angle at each bus $(V, \theta)$. 


\section{$4 \quad$ Case Study}

\subsection{Test system description}

In order to illustrate de exploitation of the tool that can be used for active and reactive power scheduling and voltage/var control in autonomous MG, the test system from [3] was adopted. This MG test system - LV grid (400 V nominal voltage) - includes 3 VSI, 17 buses, 13 loads and 8 PV generators (Fig. 5). The lines of the MG test system are predominantly resistive ( $\mathrm{R} / \mathrm{X}$ ratio in the range of 3 to 5), as it is a typical characteristic of LV distribution grids. In the overall procedure that is proposed, a three-phase balanced operation of the system is assumed.

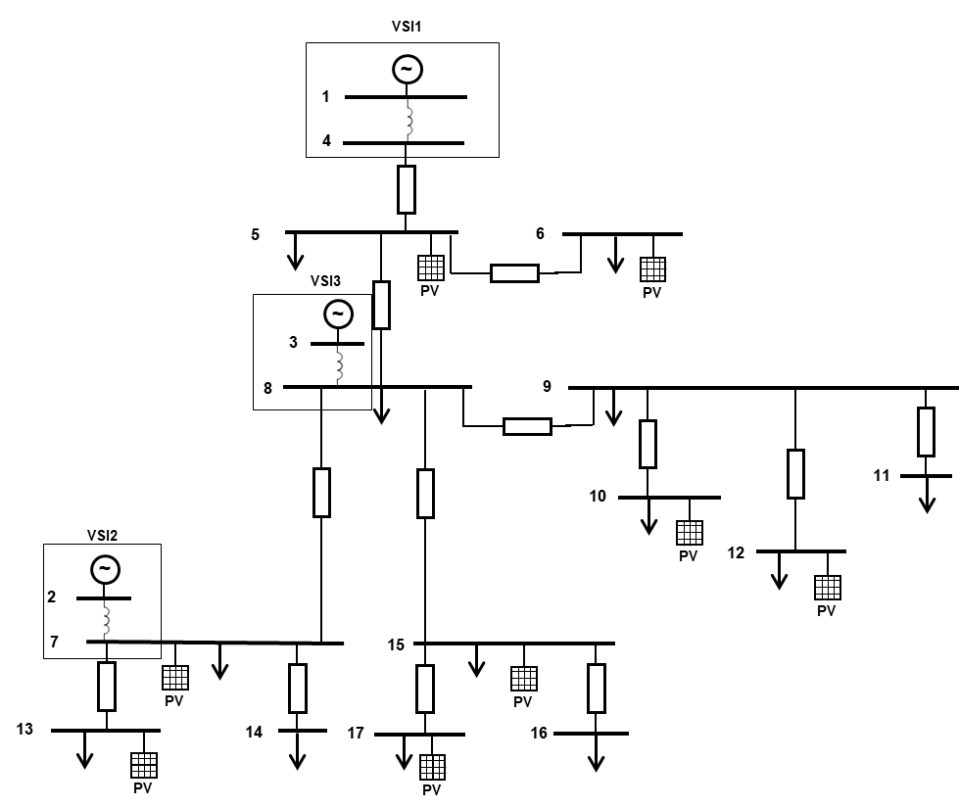

Fig. 5. MG test system single-line diagram.

The admissible range for the buses voltage magnitude is presented in Table $\mathbf{1}$.

Table 1. Admissible bus voltage range

\begin{tabular}{ccc}
\hline Bus & Vmin (p.u.) & Vmax (p.u.) \\
\hline $\mathbf{1 - 3}$ & 0,90 & 1,10 \\
$\mathbf{4 - 1 7}$ & 0,95 & 1,10 \\
\hline
\end{tabular}

The active and reactive power range for all the power converters (VSI) is presented in Table 2. The charging and discharging efficiency considered for the energy storage devices is $80 \%$ (a typical value), which takes into account not only the efficiency of the battery in itself but also the efficiency of the inverters and the energy wasted in the 
auxiliary services [10]. The maximum storage capacity of each VSI is exhibited in Table 3 , as well as the initial value assumed for the $\mathrm{SoC}$ of each unit. In addition, the SoC's lower limit is assumed to be $20 \%$ of the unit nominal energy capacity.

Table 2. Active and reactive power range in VSI

\begin{tabular}{ccccc}
\hline VSI & Pmax $(\mathbf{k W})$ & Qmax $(\mathbf{k v a r})$ & Pmin $(\mathbf{k W})$ & Qmin $(\mathbf{k v a r})$ \\
\hline $\mathbf{1}$ & 200,0 & 130,0 & $-200,0$ & $-130,0$ \\
$\mathbf{2}$ & 100,0 & 65,0 & $-100,0$ & $-65,0$ \\
$\mathbf{3}$ & 50,0 & 32,5 & $-50,0$ & $-32,5$ \\
\hline
\end{tabular}

Table 3. Storage capacity limits and initial values.

\begin{tabular}{cccc}
\hline VSI & Max $(\mathbf{k W h})$ & Min $(\mathbf{k W h})$ & Initial SoC $(\mathbf{k W h})$ \\
\hline $\mathbf{1}$ & 210 & 42 & 105 \\
$\mathbf{2}$ & 160 & 32 & 80 \\
$\mathbf{3}$ & 120 & 24 & 60 \\
\hline
\end{tabular}

The envisioned MG integrates also solar PV generation systems. This type of source is assumed to have the capability of providing reactive power injection the grid (inductive or capacitive) within a given range (in this case, $\pm 20 \%$ of the active power). Therefore, PV generators total installed power capacity is $138 \mathrm{~kW}( \pm 28 \mathrm{kvar})$. The total peak load present in the system is $67 \mathrm{~kW}$ (active) and $15 \mathrm{kvar}$ (reactive).

It is assumed that this MG becomes isolated at a given time and that will remain in those operating conditions for 24 hours onwards. For this time interval, it is necessary to define the energy scheduling and voltage and reactive power control for the overall system. Therefore, it is assumed forecasted load and PV production profiles for the MG, which are represented in Fig. 6 (normalized values with respect to the maximum load and PV generation installed capacity previously defined).

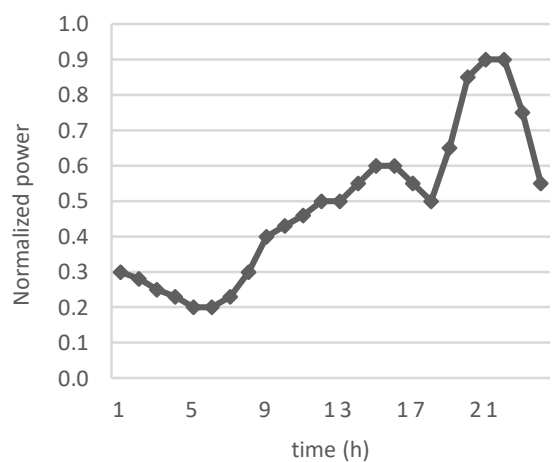

(a)

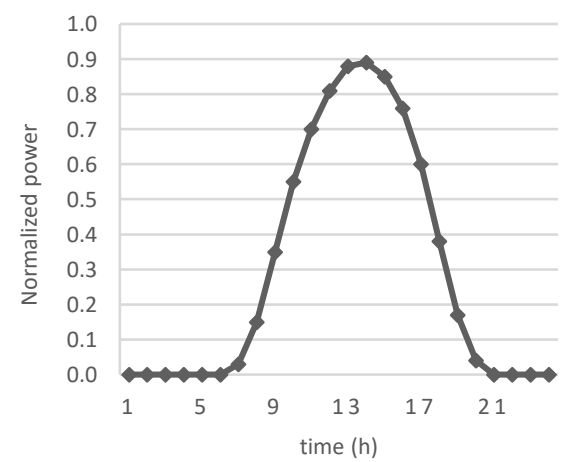

(b)

Fig. 6. Load profile forecast (a) and PV generation profile forecast (b). 
The proposed approach tool was developed using Matpower [11] and its solvers and the simulations were performed in a MATLAB ${ }^{\circledR}(\mathrm{R} 2016 \mathrm{~b})$ environment on a PC with an Intel Core i7-2600 CPU @ 3.4 GHz and 8.0 GB of RAM.

\subsection{Obtained results}

Active power scheduling. The obtained results regarding the scheduled VSI active power output and the SoC of the associated energy storage devices (Fig. 7) demonstrate that the storage devices are predominantly injecting power to the grid when there is low PV generation (from hour 1 to 9 and from hour 19 to 24) and high power consumption (from hour 20 to 22). The batteries charge mostly during the time of high sunlight exposure, which is during hour 12 until hour 19.

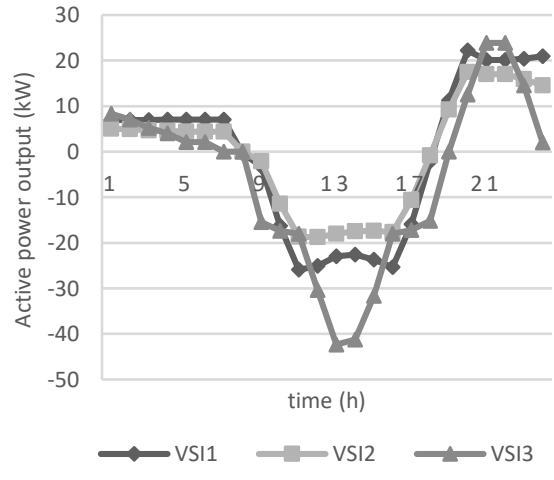

(a)

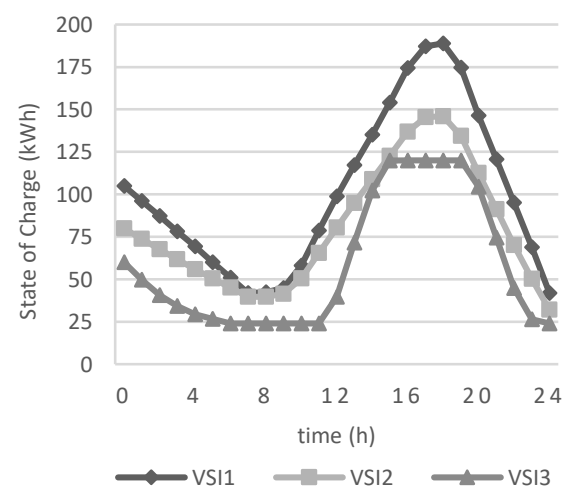

(b)

Fig. 7. VSI's active power output (kW) (a) and energy storage devices capacity (kWh) (b) evolution over time.

Reactive power scheduling. The objective function of this problem forces the PV generators and VSI to adjust their reactive power output (Fig. 8) in order to minimize voltage deviations from the nominal value (1 p.u.). 


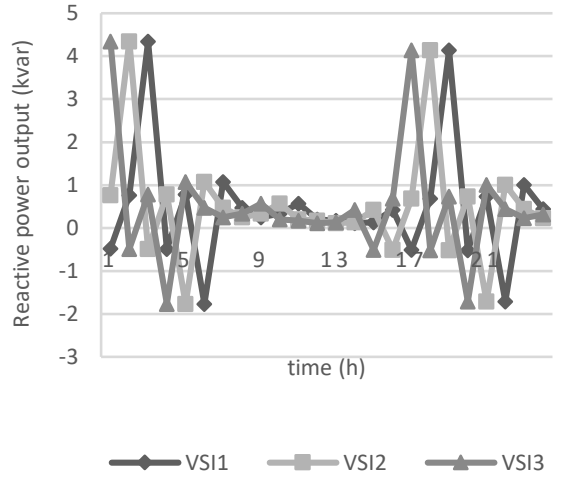

(a)

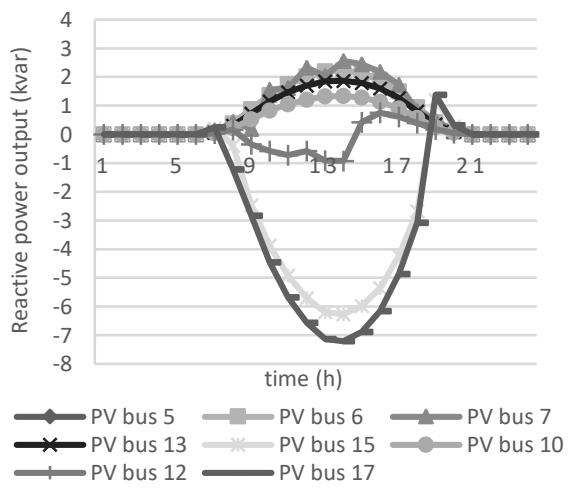

(b)

Fig. 8. Reactive power output of VSI (a) and PV generators (b).

Bus voltages. Regarding the bus voltages, it is possible to infer from Fig. 9 that there is little variation (in terms of magnitude) from the nominal value, especially from the VSI's internal bus. This is because of the OF used in this OPF. Some variability specially occurs in hours of high PV generation (maximum deviation of 5.5\% from hour 11 to 15 at bus 15 ) and peak load values (maximum deviation of $2.5 \%$ from hour 20 to 22 at bus 4$)$.

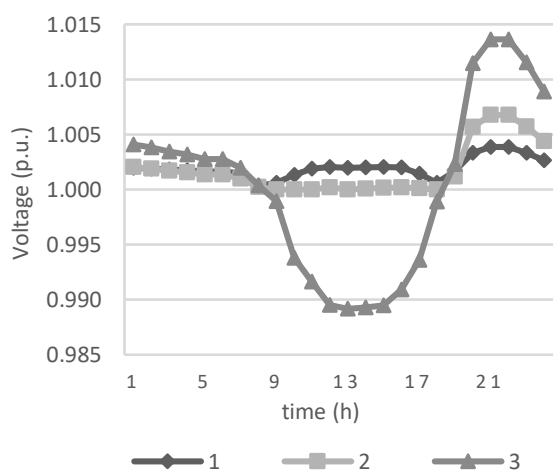

(a)

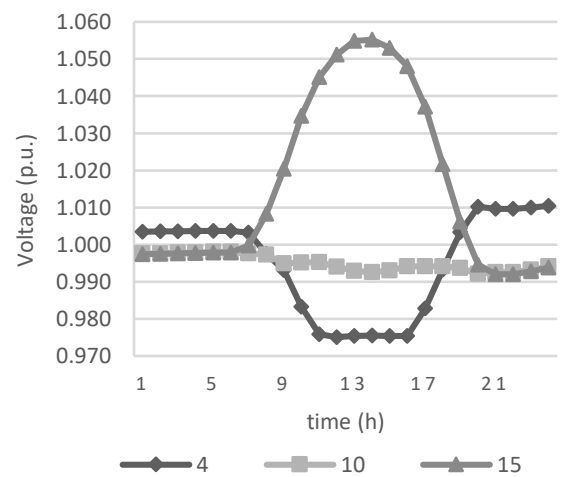

(b)

Fig. 9. Voltage magnitude (p.u.) for VSI buses (a) and buses 4, 10 and 15 (b).

Active power losses. Regarding the active power losses over time there is the evident conclusion from Fig. 10: charging and discharging the storage devices wastes more energy than the power exchanges in the feeders (because of the charging and discharging efficiencies that were considered). Regarding the grid active power losses, the reduced values that can be observed are a consequence of the relatively small power transactions and the reduced length of the cables. 


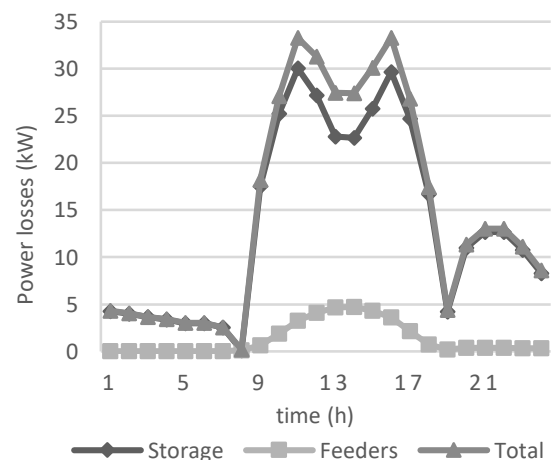

(a)

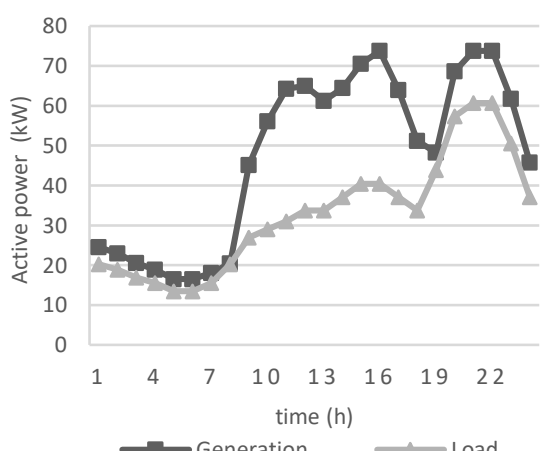

(b)

Fig. 10. Active power losses (a) vs total active power generation and load (b) (kW).

\section{$5 \quad$ Conclusions and future works}

To support the autonomous operation of a MG, this paper presents a multi-temporal approach that allows the scheduling of active and reactive power/voltage control for storage devices and DG units. This approach exploits a multi-temporal formulation since it is necessary to take into account the SoC of the storage devices. This SoC depends not only of the amount of power that is necessary to output in a specific moment but also is subjected to the energy left in store from the previous period. Additionally, the problem formulation took into consideration the specific characteristics of a droopcontrolled MG operating in islanded mode, in particular regarding the voltage and reactive power droop implemented in VSI-type converters.

After the successful validation of the proposed approach, additional work is necessary in order to consider new issues such as the unbalanced operation of the LV MG due to the presence of a significant number of single phase devices (loads and small generation systems), as well as the need of exploiting possible flexibility ranges that can be provided by resources (responsive loads) and that can have a beneficial contribution to support islanding operation of the MG system. For example, the consideration of specific strategies for the consideration of flexible loads that can be adjusted over time is a valuable feature in order to provide additional support the overall energy management problem in the islanded MG.

\section{Acknowledgments}

This work was financed by the ERDF - European Regional Development Fund through the Operational Programme for Competitiveness and Internationalization - COMPETE 2020 Programme and by National Funds through the FCT - Fundação para a Ciência e a Tecnologia (Portuguese Foundation for Science and Technology) within project "ERANETLAC/0005/2014". 


\section{References}

1. J. Simpson-Porco, Q. Shafiee, F. Dörfler, J. Vasquez, J. Guerrero and F. Bullo, "Secondary Frequency and Voltage Control of Islanded Microgrids via Distributed Averaging," IEEE Transactions on Industrial Electronics, vol. 62, no. 11, pp. 7025-7039, 2015.

2. H. Han, X. Hou, J. Yang, J. Wu, M. Su and J. Guerrero, "Review of Power Sharing Control Strategies for Islanding Operation of AC Microgrids," IEEE Transactions on Smart Grid, vol. 7, no. 1, pp. 200-215, 2016.

3. C. S. T. Gouveia, "Experimental Validation Of Microgrids:Exploiting The Role Of Plug-In Electric Vehicles, Active Load Control And Micro-Generation Units," University of Porto, Faculty of Engineering, PhD Thesis, Porto, 2014.

4. C. C. L. Moreira, "Identification and Development of Microgrids Emergency Control Procedures," University of Porto, Faculty of Engineering, PhD Thesis, Porto, 2008.

5. I. L. A. Nascimento, "Voltage and Reactive Power Control in Autonomous Microgrids," University of Porto, Faculty of Engineering, MSc Dissertation, Porto, 2017.

6. J. A. P. Lopes, C. Moreira and A. G. Madureira, "Defining Control Strategies for MicroGrids Islanded Operation," IEEE Transactions on Power Systems, vol. 21, no. 2, pp. 916-924, 2006.

7. C. Marnay, S. Chatzivasileiadis, C. Abbey, R. Iravani, G. Joos, P. Lombardi, P. Mancarella and J. Appen, "Microgrid Evolution Roadmap," in 2015 International Symposium on Smart Electric Distribution Systems and Technologies (EDST), Viena, 2015.

8. X. Wu, C. Shen and R. Iravani, "Feasible Range and Optimal Value of the Virtual Impedance for Droop-Based Control of Microgrids," IEEE Transactions on Smart Grid, vol. 8, no. 3, pp. 1242-1251, 2017.

9. J. L. Meirinhos, D. E. Rua, L. M. Carvalho and A. G. Madureira, "Multi-temporal Optimal Power Flow for voltage control in MV MVnetworks using Distributed Energy Resources," Electric Power Systems Research, vol. 146, pp. 25-32, 2017.

10. C. McKay, "Energy Storage Networks," WTWH Media LLC and its licensors, 25 January 2018. [Online]. Available: https://www.energystoragenetworks.com/three-battery-typeswork-grid-scale-energy-storage-systems/. [Accessed 1810 2018].

11. R. Zimmerman and C. Murillo-Sánchez, Matpower 6.0 User's Manual, PSerc, 2016. 